\title{
PROPOSED METHODOLOGY FOR A DATA-DRIVEN SIMULATION FOR ESTIMATING PERFORMANCE MEASURES ALONG SIGNALIZED ARTERIALS IN REAL-TIME
}

\author{
Dwayne Henclewood \\ Michael Hunter
}

\author{
School of Civil and Environmental Engineering \\ Georgia Institute of Technology \\ Atlanta, GA 30332, U.S.A.
}

\begin{abstract}
Congestion is one of the major issues facing today's transportation sector. Recent efforts have been geared toward providing traffic information to travelers, to facilitate better travel decisions, and transportation facility managers, to allow them to better manage traffic operations. Currently, real-time traffic information is primarily limited to freeways and a small subset of major arterials. This research effort explores the feasibility of an online data-driven simulation based methodology to address the lack of real-time arterial performance measures. The core of this methodology is the development of an online simulation tool that relies on commonly available arterial point sensor data, such as that from loop detectors or video cameras. Preliminary analysis indicates that the approach being considered is feasible as a model of the "real-world" was capable of reflecting performance measures with relatively high levels of accuracy. Limitations of the current research design and more immediate future directions are also presented.
\end{abstract}

\section{INTRODUCTION}

Congestion is one of the major issues facing today's transportation sector. Of the more common approaches to alleviating congestion on today's roadways is the use of Advanced Traveler Information Systems (ATIS) and Advanced Traffic Control Systems (ATCS). In an ATIS it is hoped that given relevant information regarding the current conditions of the roadway travelers will make better decisions regarding travel options. ATCS utilize real-time data to better manage traffic operations.

To support ATIS and ATCS public agencies, and more recently some private firms, outfit the roadway infrastructure, particularly urban freeways, with point sensors, e.g. video detection systems, loop detectors, and radar. The output from these sensors are then processed to determine current roadway conditions. Often, information regarding current roadway conditions is available via the

\author{
Richard Fujimoto
}

\author{
Computational Science and Engineering Division \\ Georgia Institute of Technology \\ Atlanta, GA 30332, U.S.A.
}

state's transportation department and/or some alternate source over the internet. For example, www.traffic.com provides real-time traffic information in several major US cities.

Despite the expansive traveler information available and investments in advanced control technology, there are significant limits to the quality and coverage area of such systems. Currently, real-time traffic information is primarily limited to freeways and a small subset of major arterials. An effective, real-time measure of traffic operations on the arterial network is generally not available. The extent of available arterial performance information typically available is a snap shot from video cameras at disparate locations. Most arterials also do not utilize ATCS, instead relying on more traditional control strategies.

This research effort explores the feasibility of an online data-driven simulation based methodology to address the lack of real-time arterial performance measures. At the core of this methodology is the development of a data-driven online simulation tool that relies on commonly available arterial point sensor data, such as that from loop detectors or video cameras. The following sections will present some background on the use of traffic data to estimate roadway performance measures, followed by the conceptual framework of the methodology being proposed. In addition, a proof of concept, along with preliminary results, limitations, and future directions will be presented.

\section{PROBLEM STATEMENT AND OBJECTIVE}

The overarching goal of this effort is to address the lack of available real-time arterial traffic operations performance measures. Such performance measures may be used by road users, to make better trip decisions, and transportation agencies, to aid in facility management; for instance in conjunction with advance traffic signal control systems. This additional information may be seen as a 
part of a larger effort to aid in the continuous effort to reduce congestion throughout the transportation network.

This effort currently focuses on fixed roadway sensor data, such as that obtained from inductance loop detectors and/or video detection systems. Detector data is integrated with a traffic simulation software package to model current roadway conditions in real-time. In this paper the feasibility of the methodology being introduced is explored with the aid of two traffic simulation instances, with one providing simulated "real world" detector data, and the other tracking the real world performance through the use of that data.

With this online data-driven configuration it is hoped that existing and near future travel times and other arterial performance metrics (queues, delays, etc.) may be determined and passed to travelers and transportation management agencies alike. Furthermore, in future efforts simulation clones could be instanced in real-time that may then be the executed forward in faster than real-time to generate traffic predictions.

\section{LITERATURE REVIEW}

The following section will present a brief overview of some of the many previous efforts that explored means of estimating and predicting performance on signalized arterials.

\subsection{Estimating Arterial Performance Measures}

Over the years there have been a number of research efforts examining means to estimate and predict various performance measures of signalized arterials from point sensor data. Work on determining these measures from point sensors began as early as the late 1970s with P. G. Gipps developing one of the earliest models to estimate travel time (vehicle delay) from loop detector output (Gipps 1977). Since that effort, many researchers have tried to improved upon this model using a series of advanced mathematical concept, ranging from Bayesian Theorem (Park and Lee 2004) to neural network models (Liu, van Lint and van Zuylen 2007).

The work presented in (Sisiopiku and Rouphail 1994) provides a comprehensive overview of many early models that aimed at using detector output to estimate arterial performance measures. In reviewing and comparing many of these models, the authors highlight some of the general limitations. Some of these limitations were also echoed in (Liu, van Lint, and van Zuylen 2007). Despite the fact that simulation results are in general promising, limitations were found including a lack of transferability as the models are very link-specific and site-dependent, inadequate accounting for differences in travel time due to movement type and a lack of acceptable field evaluations.
A further review of similar models for estimating and predicting arterial performance measures was presented in (Zhang and Kwon 1997). The focus of this article was to largely compare models that estimate travel time on urban arterials using loop detectors. The authors grouped these models into five categories. These categories are as follows: (1) regression, (2) dynamic input-output, (3) pattern matching, (4) sandglass and (5) models developed by the Bureau of Public Roads (BPR).

Upon examining the above arterial travel-time estimation models one recognizes that despite their on-line, real-time potential, which has not been realized, these models were not able to be implemented in the field either due to their input data requirements or unsatisfactory test performance. The goal of this research is to build upon these efforts and overcome these issues through the use of a real-time data-driven simulation approach. Such a system should be able to use input data from loop detectors and/or video detection systems to estimate and predict, in real-time, various arterial performance measures including travel time. (BPR 1964; Gault and Taylor 1977; Gipps 1977; Bohnke and Pfannerstill 1986; Usami, Ikenoue, and Miyasako 1986; Zhang and Kwon 1997)

\section{METHODOLOGY AND PROOF OF CONCEPT}

As stated the goal of this research effort is to use real world sensor data to drive a traffic simulation, with the aim of mirroring the current conditions on an arterial roadway. After successfully capturing current conditions, future performance may then be predicted by exporting system states from the real-time roadway simulation to client simulation models that run at faster than real-time. To further illustrate the conceptual framework of this approach see Figure 1.

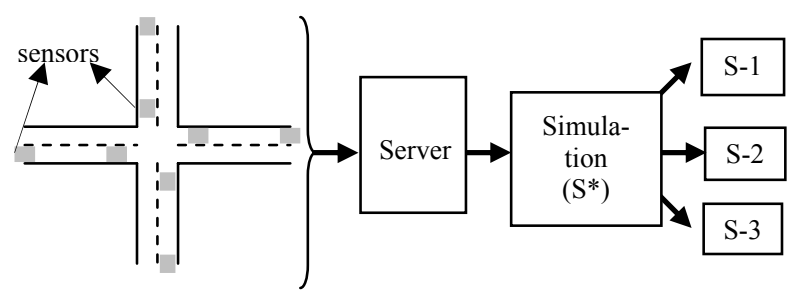

Figure 1: Conceptual Framework

In the above conceptual framework the Server is responsible for polling and processing the data received from all the point sensors at a predetermined time interval of, say, one second. The real-time arterial traffic simulation $\left(\mathrm{S}^{*}\right)$ subscribes to the data on the server for the sensors within the area being modeled. Once the data is processed in the server it is transmitted to the subscribing traffic simulation clients that then use the data to drive traffic flow. In the framework depicted in Figure 1, S* aims to provide a real-time mirror image of the roadway's 
current real-world conditions. From $\mathrm{S}^{*}$ states saves of the current conditions may then be taken and used in client simulations S-1, S-2, S-3 etc. to provide various possible future scenario analysis. Results from these clients may be utilized to provide users with estimated current and future travel times, delays and other relevant roadway performance measures.

To implement this framework the research team envisions a series of steps that will not only provide the foundation for future work but also provide key lessons on the path to successful delivery of current and future roadway conditions in real time. These steps include a proof of concept test (the primary focus of this paper), a hardwarein-the-loop scenario and a field test.

\subsection{Proof of Concept}

The proof of concept seeks to provide insight into whether or not the approach being considered is in fact feasible. This experiment will use two VISSIM simulation instances. VISSIM is an off-the-shelf microscopic, time step and behavior-based multi-purpose traffic simulation program (PTV 2006). One instance will represent the "real-world" the other will attempt to simulate the real-world in real time, hereafter referred to as the "modeled-world". The inputs to the real-world model include traffic volumes over a 4-hour period (reflective of a peak period), signal timing data, and vehicle turning movements. The modeled-world simulation has the same roadway configuration, signal timing data and historical turning movement percentages equal to the four hour average of the real-world model. A key point to note about the modeled-world simulation is that it is not given any vehicular volumes. Vehicles will be generated according to the data obtained from the detectors in the real-world simulation instance.

The aim of this exercise to successfully approximate roadway conditions of the real-world simulation in the modeled-world simulation. To determine how well the modeled-world replicates the real-world, several roadway performance measure will be collected and compared. Such performance measures include travel time and delay over representative paths and queue lengths at the approaches of the various intersections.

\subsubsection{Experimental Design}

A three intersection arterial was created using VISSIM, with each intersection under two-phase semi-actuated signal control. Each roadway is a two-way arterial, with one lane in each direction. A loop detector is placed 30 meters from the upstream end of each entrance link, for a total of eight boundary loop detectors. These detectors, used only in the real-world simulation model, are responsible for capturing the presence of a vehicle and its speed, as a ve- hicle enters the network. There are 6 additional detectors, one on each intersection cross street approach, in both the real- and modeled-world. These detector are used to implement the semi-actuated traffic signal control, with no data currently passed from these detectors to the modeledworld simulation. Both models simulate a 4 hour time period during which the maximum network volume reached is approximately 1200 vehicles/hour and a minimum of approximately 550 vehicles/hour.

A framework implemented in $\mathrm{C}++$ was developed to implement the system shown in Figure 1. Using this framework, VISSIM provides a direct means of interacting with a simulation during runtime through its COM module. When establishing communication between the two simulation models a unidirectional named pipe was created. A pipe is a specific section of memory that is used for the purposes of communicating between a server and one or more clients. As for the difference between a server and a client as it relates to pipes, the server, better known as a pipe-server, is the process that creates the pipe, and the client, or pipe-client, is the process that connects to the created pipe (Microsoft 2008). The named pipe that was created was fashioned so that the real-world simulation model was essentially the pipe-server, which was only able to write to the pipe. The pipe-client was the modeled-world simulation and it was only able to read from the pipe. Pipes are capable of two way communication but for the purposes of this experiment a unidirectional pipe was sufficient.

The pipe-server, connected to the real-world simulation model, polls each of the eight detectors that are placed at the edge of the network to collect vehicle speed, location and lane data once every simulation second. In this example, given the fixed detector locations, a detector ID would also be sufficient in place of the location and lane data, however, passing location and lane data was undertaken as this allows for more robust data streams in future experimental iterations. At the end of each second the pipe server writes an [8] x [3] array to the pipe containing the detector information over the last second. The array is then read by the pipe-client and the information is implemented in the modeled-world simulation. For a graphical representation of the experimental design, see Figure 2. 


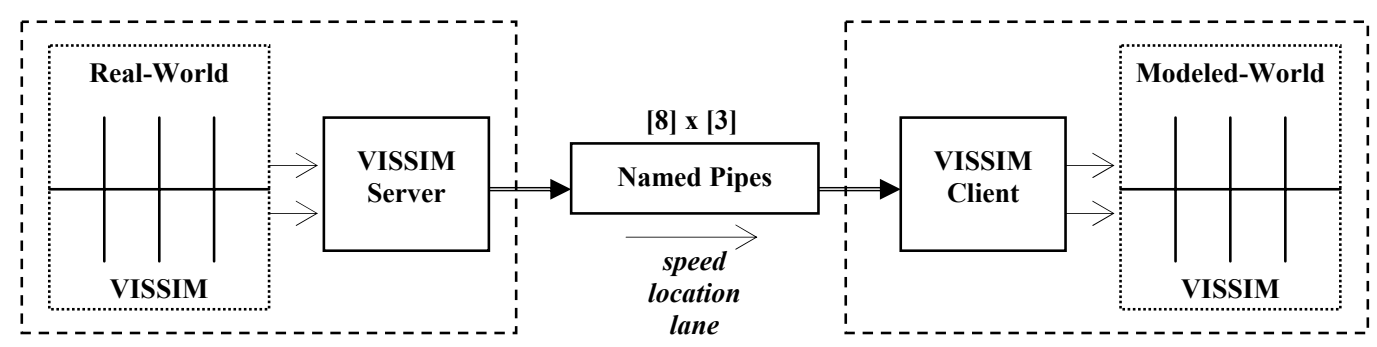

Figure 2: Experimental Design for Proof of Concept

In this design, reliable, ordered communications are assumed. This aspect of the proof of concept experiment was assumed to ensure that the modeled-world simulation remains in sync with that of the real-world. Recall that the pipe is unidirectional, with the pipe-server writing to the pipe every second and the pipe-client reading every second. Using this knowledge the execution speed of the model world is driven by the real world, with the modeled-world executing a simulation second only when a second of data is received from the real-world data server. Therefore if the pipe-server runs ahead of the pipe-client, say by 5 seconds, due to delayed processing by the pipeclient, it would have written 5 [8]x[3] arrays to the pipe. The named pipe used in this experiment operated on a first-in-first-out (FIFO) basis and as a result the pipeclient will then read those 5 arrays, and advance its simulation clock in five one second increments, at maximum processing speed. When the pipe is empty the pipe-client, along with its simulation, will then pause until the pipeserver writes another array to the pipe. Future versions of the framework will integrate timestamps directly into the data stream and develop data consistence checks. To further illustrate the structure of the server - client relationship the, see the following pseudo-code.

\section{Pipe-server (Real-World)}

$$
\begin{aligned}
& \text { for }(i=0, i<=\text { simulation period, } i++) \\
& \{\text { advance simulation } 1 \mathrm{sec} \\
& \text { read vehicle speeds from the } 8 \text { detectors } \\
& \text { write [8]x[3] to pipe \} }
\end{aligned}
$$

\section{Pipe-client (Modeled-World)}

$$
\begin{aligned}
\text { for }(i= & 0, i<=\text { simulation period, } i++) \\
& \text { read [8]x[3] from pipe } \\
& \text { input vehicle speeds into simulation } \\
& \text { advance simulation } 1 \text { sec }\}
\end{aligned}
$$

\subsubsection{Simulated Time Frame}

A four hour simulation time period was used, capturing the transition into and out of the peak period. At the end of the simulation period the average travel times and delays from 7 representative paths, along with the queue lengths at each intersection approach was collected from both the real-world and modeled-world simulations. These performance measures are presented in 10-minute interval aggregations.

\subsubsection{Scenarios}

The results from two scenarios are presented. Scenario 1 relies on the assumption of ideal detector performance, with every real-world vehicle and its associated speed accurately detected and passed to the modeled-world. Under such an assumption the primary difference between the real-world and modeled-world results will be due to randomness in driver and vehicle characteristics and potentially different path selection decisions of a vehicle in the real-world and its simulated counterpart in the modeled-world.

Scenario 2 attempts to introduce more of the variability that would be expected in a field implementation. To accomplish this the research team allowed the detectors to fail to detect a vehicle with a frequency of approximately $2 \%$. Additionally, the detected speeds were allowed to vary randomly higher or lower by up to $10 \%$ of the actual vehicle speed. It is known that different point sensors have different failure rates as well as different levels of accuracy when determining vehicle speeds. Scenario 2 attempts to reflect the impact of realistic detector performance characteristics on the accuracy of the modeledworld results.

In both scenarios the vehicle speed measured over the detector in the real-world (as stated in scenario 2 these speeds may have up to a $10 \%$ error) is used as the desired vehicle speed for the vehicle placed in the modeledworld. However, if the vehicle speed was lower than the assumed range of desired speeds ( 48 to $58 \mathrm{kph}$ ) it is fur- 
ther assumed the vehicle is within congested conditions and the desired speed is randomly set within the preceding desired speed range. In this instance the vehicle is placed in the modeled-world at the highest speed possible given traffic conditions without exceeding the desired speed. If the vehicle is traveling more slowly than the desired speed it will attempt to accelerate to the desired speed as quickly as possible.

\section{RESULTS AND ANALYSIS}

Five replicate runs (R-1 through R-5) were performed with each run using a different set of random seeds for real-world and modeled-world simulation instances. Each replicate run consisted of a modeled-world being driven by the detector data of the real-world simulation, allowing for a direct comparison of real-world replicate $i$ to modeled-world replicate $i$.

Travel time and delay results for 7 paths and queue lengths for 3 approaches were compared between the real and modeled-world simulation instances for the 2 scenarios. Figure 3 and Table 1 present the naming conventions of the links in the network and the performance measure links considered. All links in the network are single lane.

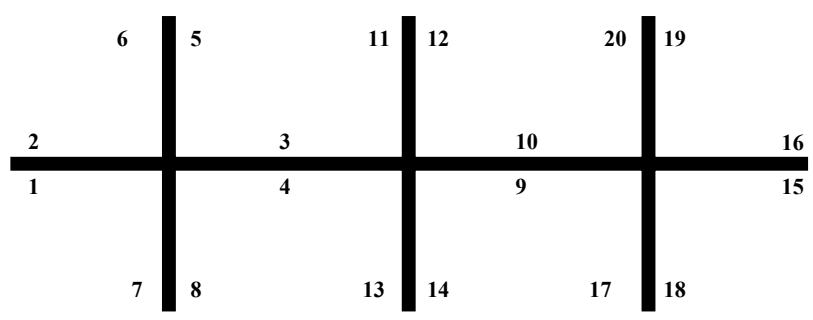

Figure 3: Roadway Network and Link Names

Table 1: Description of Performance Measures

\begin{tabular}{lccc}
\hline \multicolumn{2}{c}{ Measures } & Links & Distance $(\mathrm{m})$ \\
\hline Travel Time & Delay & & \\
\hline TT-1 & DL-1 & $1-4-9-15$ & 1308 \\
T-2 & DL-2 & $16-10-3-2$ & 1309 \\
TT-5 & DL-5 & $11-13$ & 290 \\
TT-8 & DL-8 & $4-12$ & 366 \\
TT-9 & DL-9 & $10-13$ & 382 \\
TT-10 & DL-10 & $4-9$ & 381 \\
TT-11 & DL-11 & $10-3$ & 383 \\
\hline \multicolumn{7}{r}{ Queue Length } & & - \\
\hline QL-1 & & 1 & - \\
QL-6 & & 14 & - \\
QL-7 & & 10 & \\
\hline
\end{tabular}

\subsection{Individual Performance Measures}

For most of the monitored travel time paths, delays, and queue lengths; the ideal and realistic detector modeledworld scenarios captured the performance of the real- world accurately. For example, consider Figure 4 and Figure 5 which presents the values of TT-2, from replication 2 (R-2), and of QL-6, from replication 3 (R-3), respectively, over the 4-hour simulated time period. The modeled-world in both scenarios is able to reasonably track performance measures of the real-world through the 4 hour period.

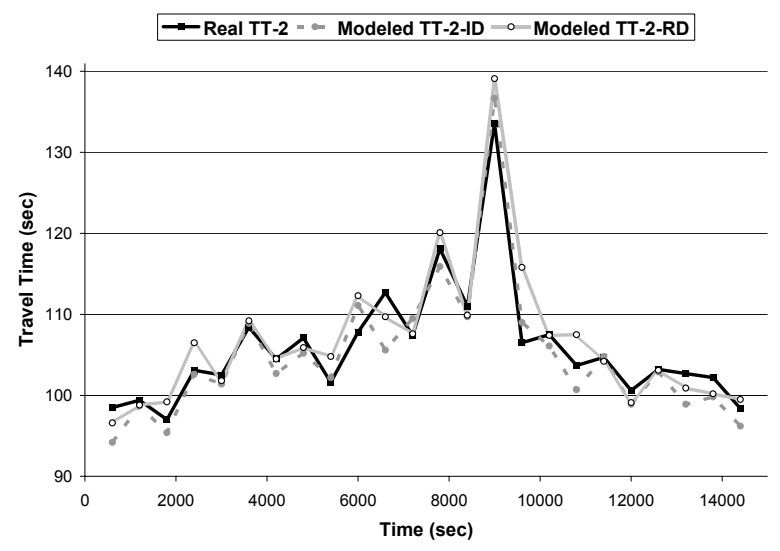

Figure 4: Average Travel Time - 2, from R-2

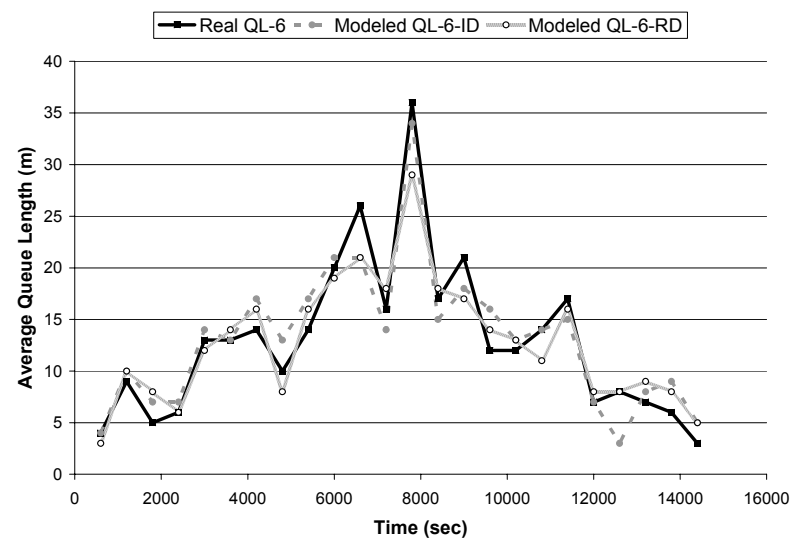

Figure 5: Average Queue Length - 6, from R-3

As seen in Figure 4 and Figure 5 the absolute differences amongst the measures from the various scenarios analyzed are minimal. From Figure 4, the average and standard deviation of the difference between the values of TT-2, from scenarios 2 and 3, were 2.06 and 1.55 seconds; and 2.10 and 2.12 seconds, respectively. Similarly, from Figure 5, the average and standard deviation of the difference between the values of QL- 6 from the two scenarios were 1.96 and 1.46 car-lengths; and 1.96 and 1.57 car lengths, respectively.

However, when considering all replicated experiment instances it was found that the model-world did not consistently track the real-world. For instance, consider Figures 6 and 7, which represents TT-1 from R-4 and DL-1 from $\mathrm{R}-3$, respectively. There is a large discrepancy in the 
estimates of these particular performance measures between 8000 and 11000 seconds, the highest demand period of the simulated time frame. In Figure 6 the average for TT-1 is approximately $73 \%$ less in the modeled-world than in the real-world, for both scenarios. And in Figure 7 , the delay measure is on average $44 \%$ lower in the modeled-world than in the real-world.

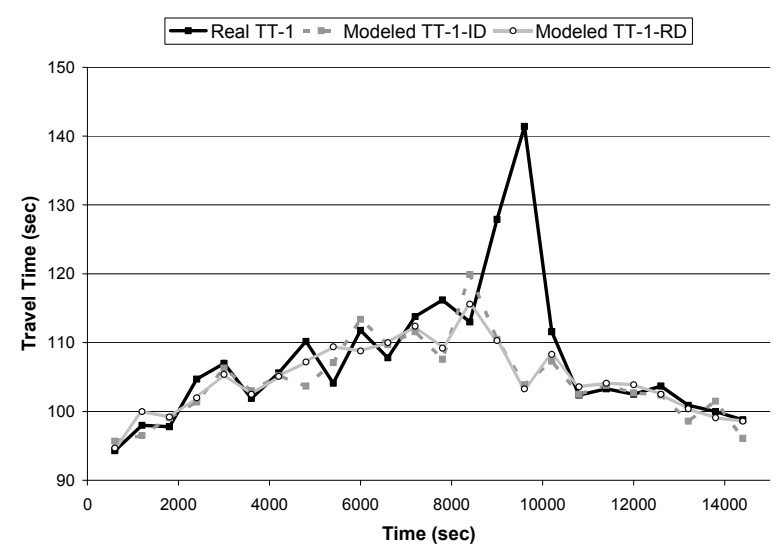

Figure 6: Average Travel Time - 1, from R-4

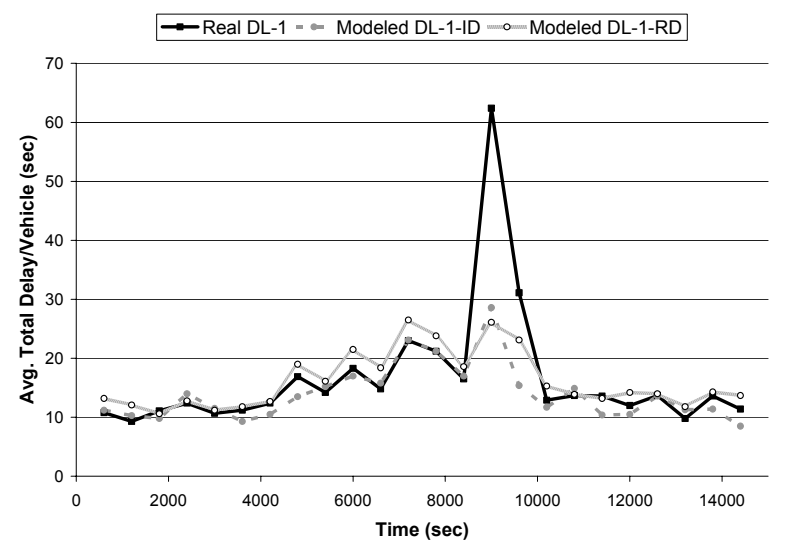

Figure 7: Average Delay -1, from R-3

The source of these differences is likely derived from two primary causes: 1) randomness in vehicle routing and 2) a smoothing of flows in the model-world. In these experiments the randomness in vehicle routing is limited to the selection of a vehicle's turning movement at an intersection. Of primary concern is the selection between through and left turn movements. For example, the intersection midway through the arterial has the highest left turn movement percentage at $16 \%$ in each direction. The randomness in left turn movements manifests itself through which vehicles in a particular platoon will make the left turn. The left turn vehicle placement in the queue can dramatically impact operations as flows approach capacity, particular in this study network as a left turning vehicle waiting for a gap will block all following (left, through, or right turning) vehicles. For example, if the $1^{\text {st }}$ vehicle in a platoon is attempting to negotiate a left turn at the arterial's middle intersection and is unable to do so the waiting delay is incurred not only for the turning vehicle but also for those vehicles queued behind the turning vehicle. Should the last vehicle in the platoon attempt to make a left turn any delay while waiting for a gap will be experienced only by the left turn vehicle.

This particular source of error cannot be addressed by boundary point sensors without knowledge of every realworld vehicle's desired path through the network. The currently data-driven simulation is based on the hypothesis that such data is likely to be unavailable, at least in the near future. However, detector data from internal network detectors may provide a means to address this issue. For example, a mainline detector at the stop bar could be used to identify when vehicles are not moving during a green phase and this information could be passed to the modeled-world simulation. Clearly, the use of internal network detector information will be one direction of future research efforts.

The second issue, a smoothing of flows in the modeled-world, has the potential to "smooth" out traffic fluctuations. Currently, irrespective of the headway with which cars enter the real-world, the modeled-world implementation algorithm has the effect of rounding the headway to the nearest second. This is particularly noteworthy for actuated traffic control, where a few tenths of a second can be the difference between a signal gaping out and a car receiving an extension of the green phase. For example, in the replicate runs where the divergence in travel time was seen at the middle intersection it was also noted that the side streets tended to receive slightly more green time. Overall this would decrease the time given to the mainline and decrease the modeled-world delays. Headway smoothing of the entering flows is a likely explanation of the extended side street green time. Future efforts will consider methods to eliminate this unintended bias.

\subsection{Consistency of Results}

The consistency of the performance measures across replicate runs was explored by calculating the difference between the real-world and scenario performance measure for each replicate trial and then averaging over the 5 replicates. Figures 8 and 9 illustrate the concept of stability using average differences in queue lengths from scenario 1 and travel times from scenario 2 , respectively. 


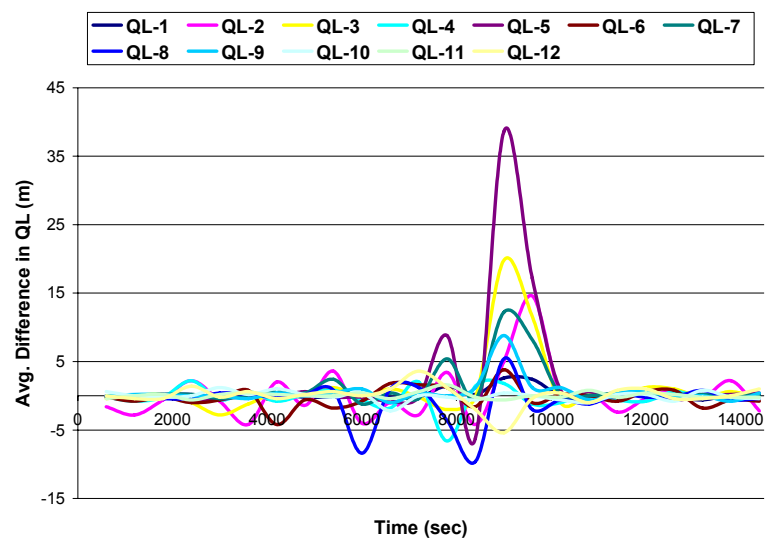

Figure 8: Average Difference in QL, Scenario1

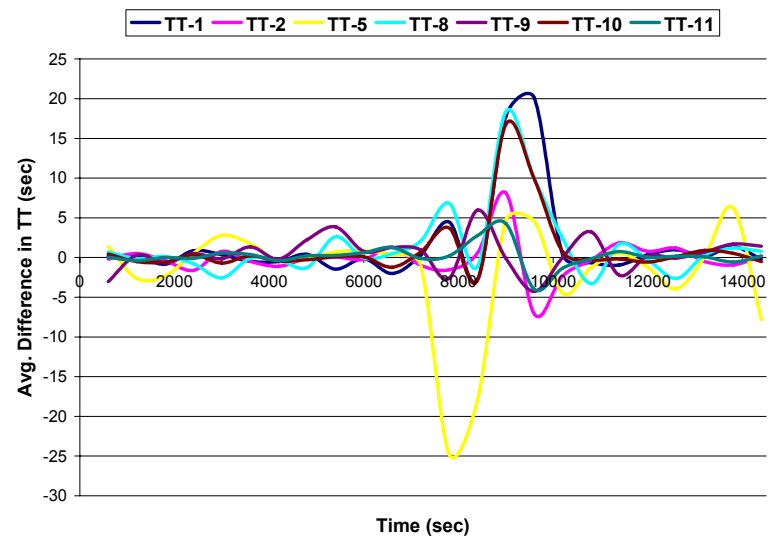

Figure 9: Average Difference in TT, Scenario 2

The above figures indicate that the methodology being considered is rather stable except for a few performance measures whose differences drastically increase during the period being considered. The reason for these difference is discussed in the previous section and will be the focus of future efforts. Overall, the modeled-world is generally successful at replicating performance measures of the real-world and is also somewhat resilient to reasonable detection errors, that is, drastically faulty data or complete detector failure is not considered in this analysis.

\section{LIMITATIONS AND FUTURE DIRECTIONS}

As seen the results indicate that under ideal and realistic detector characteristic the modeled-world provides a reasonable replication, with some notable variation, of the real-world. However, significant limitations remain in the experimental design that will be addressed in the next phase of the research.

Upon designing the proof of concept experiment, the research team limited the information passed from the real-world simulation to the information that could be actually obtained in a field implementation. The modeled- world was not provided with more information than what may be detected on today's roadways. However, in VISSIM there are approximately 12 noteworthy parameters that are used for the purposes of calibrating traffic simulation models. Table 2 lists these parameters. (PTV 2006) In the case of the presented experiments these 12 parameters are in fact equivalent, making the modeledworld simulation instance "perfectly" calibrated to that of the real-world.

Table 2: Description of VISSIM Calibration Parameters

\begin{tabular}{ll}
\hline \multicolumn{2}{c}{ Parameters } \\
\hline & \\
Emergency stopping distance & Minimum headway \\
Lane changing distance & Desired safety distance parameters \\
No. observed preceding vehicles & Maximum deceleration \\
Maximum look ahead distance & $-1 \mathrm{~m} / \mathrm{s}^{\wedge} 2$ per distance \\
Average stand still distance & Accepted deceleration \\
Waiting time before diffusion & Distance of standing and $50 \mathrm{~km} / \mathrm{h}$ \\
\hline
\end{tabular}

A key aspect of future efforts will be to explore the impact of these calibration parameters and other sources of randomness in the simulation. While perfect calibration is not possible the ability to conduct real time calibration using probe vehicle data will be explored. It is hoped that is using near-real time probe vehicle data will all for adjusting the calibration parameters such that a near-optimal fit may be obtained.

Next research steps will also include identifying, quantifying, and addressing the factors that resulted in the significant variation noted during the peak demand period. Of primary interest will be two issues discussed in section 5.1 however other possible sources for the variation will also be sought.

Finally, the current model is limited to detection at boundary points of the model. Future work will seek the incorporation of detection data from internal detectors into the model calibration. This will consider standard detections (i.e. typical actuated control layouts) and the possible of new detector placement specifically designed to aid a real-time simulation.

\section{CONCLUSION}

This paper introduced a methodology to develop a datadriven online simulation tool to deliver real-time and near future arterial performance measures to the traveling public and transportation facility managers with the aid of microscopic traffic simulation. The major objective of this paper was to demonstrate the feasibility of such as realtime simulation. As such, a proof of concept experiment was designed to have one microscopic traffic simulation instance reflect performance measures of another. This experiment was accomplished through the use of two 
VISSIM simulation instances, where one represented the real-world and the other, the modeled-world.

The aim of this effort was for the modeled-world to reflect the performance measures of the real-world, by use of a data stream of vehicle information being passed from the real-world simulation instance to that of the modeledworld. The results from this experiment demonstrated that the modeled-world is capable of reflecting the performances measures of the real-world with a relatively high level of accuracy. However, some notable discrepancies were seen. Despite the current discrepancies and limitations of the experimental design, the results presented supports the likely feasibility of this approach and future efforts on the proposed methodology.

\section{REFERENCES}

Akiva, M. B., M. Bierlaire, H. N. Kousopoulos, and R. Mishalani. 2000. Real-Time Simulation of Traffic Demand-Supply Interactions with DynaMIT: 19.

Bohnke, P., and E. Pfannerstill. 1986. A System for Automatic Surveillance of Traffic Situation. Institute of Transportation Engineers 5(1): 5.

BPR 1964. Traffic Assignment Manual Washington, DC., Bureau of Public Roads, US Department of Commerce.

Gault, H. E., and I. G. Taylor. 1977. The Use of Vehicle Detector to Assess Delay in Computer-Controlled Area Traffic Control. Newcastle, England, Transport Operation Research Group, University of Newcastle upon Tyne.

Gipps, P. G. 1977. The Estimation of a Measure of Vehicle Delay from Detector Output. Newcastle, England, Transport Operation Research Group, University of Newcastle upon Tyne.

Lin, W.-H., A. Kulkarni, and P. Mirchandani 2004. Short Term Arterial Travel Time Prediction for Advanced Traveler Information System. Intelligent Transportation Systems 8: 143-154.

Liu, H., H. van Lint, and H. van Zuylen. 2007. NeuralNetwork-Based Traffic Flow Model for Urban Arterial Travel Time Prediction. TRB 86th Annual Meeting Compendium of Papers CD-ROM.

Mahmassani, H. 2007. Development and Testing of a Real-Time Traffic Estimation and Prediction System. Available via <http://www.mti.umd.edu/ people/mahmassani/research/Research descriptions_HSM_website_10\%2004.p df $>$ [accessed September 15, 2007].

Microsoft 2008. Microsoft Developer Network (MSDN).

Park, T., and S. Lee. 2004. A Bayesian Approach for Estimating Link Travel Time on Urban Arterial Road Network. Computational Science and Its Applications-ICCSA 2004 3043/2004: 1017-1025.
PTV 2006. VISSIM 4.20 User Manual. Karlsruhe, Germany, Planung Transport Verkehr AG.

RITA 2008. Vehicle Infrastructure Integration (VII), Research and Innovative Technology Administration (RITA), U.S. Department of Transportation (US DOT).

Sisiopiku, V. P., and N. M. Rouphail. 1994. Toward the Use of Detector Output for Arterial Link Travel Time Estimation: A Literature Review. Transportation Research Record 1457: 158-165.

Usami, T., K. Ikenoue, and T. Miyasako 1986. Travel Time Prediction Algorithm and Signal Operations at Critical Intersection for Controlling Travel Time. Second International Conference of Road Traffic Control, Institute of Electrical and Electronics Engineers.

USDOT 2006. Intelligent Transportation Systems, United States Department of Transportation.

Yang, J.-S. 2006. A Non-linear State Space Approach to Arterial Travel Time Prediction, Minnesota Department of Transportation, Office of Research Services.

Zhang, H. M., and E. Kwon. 1997. Travel Time Estimation on Urban Arterials Using Loop Detector Data. Iowa City, IA, Public Policy Center.

\section{AUTHOR BIOGRAPHIES}

DWAYNE HENCLEWOOD is a Ph.D. student at the Georgia Institute of Technology. His current research interest is in arterial performance evaluation and microscopic traffic simulation. He holds a M.S. in Civil Engineering from the University of Massachusetts Amherst. His email address is $<$ dahencle@gatech.edu>.

MICHAEL HUNTER is an assistant professor in the School of Civil and Environmental Department at the Georgia Institute of Technology. His primary areas of interest are traffic safety, operational performance and control, and simulation. He earned his Ph.D. at the University of Texas at Austin in August 2003. His e-mail address is $<$ michael.hunter@ce.gatech.edu>.

RICHARD FUJIMOTO is a regents' professor and chair of the Computational Science and Engineering Division at the Georgia Institute of Technology. He received his Ph.D. and M.S. degrees from the University of

California (Berkeley) in 1980 and 1983. Among his past activities he lead the definition of the time management services for the DoD High Level Architecture (HLA), and has also been a member of the steering committee for the Workshop on Parallel and Distributed Simulation, (PADS) since 1990. His email address is <fujimoto@cc.gatech.edu>. 